\section{Beatmung und ARDS: Gibt es Parameter zur frühen Risikoabschät- zung?}

Neto AS et al. Epidemiological characteristics, practice of ventilation, and clinical outcome in patients at risk of acute respiratory distress syndrome in intensive care units from 16 countries (PRoVENT): an international, multicenter, prospective study. Lancet Respir Med 2016; 4 : $882-893$

Rund $4 \%$ aller Beatmungspatienten entwickelten ein ARDS. Initiale respiratorische Parameter wie PEEP und Oxygenierung indizieren dieses Risiko nicht eindeutig und sind deshalb nicht zur Risikostratifizierung geeignet.

Bei beatmeten Patienten besteht immer das Risiko ein ARDS auszuprägen. Die spezifischen, beatmungsassoziierten Risiken hierfür sind nicht bekannt. Kritische Beatmungskonstellationen zu erkennen ist aber von hoher Relevanz. Hieraus ließe sich eine individuell adaptierte, respiratorische ARDS-Prophylaxe ableiten.

Die internationale Multizenterstudie (PRoVENT) erfasste die Beatmungspatienten von 190 Intensivstationen. Diese Daten nutzten die Autoren für ihre Subanalyse. Sie identifizierten Patienten, die in einem definierten Zeitraum (7 Tage) längerfristig beatmet werden mussten. Bei Studienbeginn quantifizierten sie das klinische ARDS-Risiko anhand des Lung Injury Prediction Score (LIPS). Der Schwellenwert lag hier bei vier ScorePunkten. Diese Einschätzung wurde nicht systematisch in die individuelle Beatmungsstrategie einbezogen. Patienten, die bereits zu Studienbeginn ein ARDS entwickelt hatten, erkannten die Forscher anhand der Berlin-Definition und bezogen diese Patienten nicht in die Studie ein.

Primäres Ziel der Studie war die Erfassung der ARDS-Risiko-Inzidenz in Abhängigkeit von der klinischen Ausgangslage, den initial gewählten respiratorischen Parametern und demografischen Gegebenheiten. Als sekundäres Outcome ermittelten die Forscher die Rate der ARDS-Manifestationen. Sie berücksichtigten zusätzlich demografische und epidemiologische Aspekte als mögliche Kofaktoren.

\section{Ergebnisse}

Bei $4 \%$ der gesamten Beatmungspatienten kam es zum ARDS. $30 \%$ aller Beatmeten wiesen ein klinisch relevantes ARDSRisiko auf. $7 \%$ hiervon entwickelten diese Komplikation. Patienten ohne vorbestehendes Risiko dagegen waren nur zu 3\% betroffen. Die Autoren errechneten eine ARDS-Risiko-Inzidenz von 0,14 Fällen pro Beatmungsfall pro Woche.

Die respiratorischen Parameter zu Studienbeginn zeigten keine relevante Risikoassoziation. Zwar lag der anfänglich gewählte PEEP bei ARDS-Risiko gering- gradig höher ( 6 vs. $5 \mathrm{mmHg}$ ). Auffallend war auch, dass diese Patienten anfangs mit einer gering verminderten Oxygenierung imponierten $\left(\mathrm{F}_{\mathrm{i}} \mathrm{O} 2\right.$ 0,6 vs. 0,5). Diese Befunde bewerteten die Autoren aber nicht als richtungsweisend. Die übrigen Beatmungsparameter (z.B. Tidalvolumen) standen in keinem Risikozusammenhang.

Unklar bleibt für die Autoren, warum demografische Faktoren das Risiko der Beatmungspatienten modifizieren. So variierte die ARDS-Risiko-Inzidenz in Abhängigkeit vom Studienort erheblich (Nordamerika $18 \%$, Südamerika 43\%). Die Autoren vermuten, dass hier möglicherweise zeitliche Effekte (z.B. Grippesaison) oder divergierende klinische Algorithmen maßgeblich sind.

FAZIT

Erste epidemiologische Daten lassen vermuten, dass das ARDS-Risiko von Beatmungspatienten frühzeitig, anhand der Beatmungsparameter, identifiziert werden kann. Die hierzu vorliegenden Befunde sind, so die Autoren, nur als Indiz zu werten. Weitergehende Forschungen zur Beschreibung von validen respiratorischen Risikoparametern erscheinen demnach gerechtfertigt.

Dr. med. Horst Gross, Berlin 